\title{
The thermal conductivity of periodic particulate composites as obtained from a crystallographic mode of particle packing
}

J. Venetis: (Cor. Author), School of Applied Mathematics and Physical Sciences NTUA, Email: johnvenetis4@gmail.com

E. Sideridis: School of Applied Mathematics and Physical Sciences NTUA, Section of Mechanics, 5 Heroes of Polytechnion Avenue, GR - 15773 Athens, Greece.

Telephone: +302107721224 Fax: +302107721302

Email: siderem@central.ntua.gr

\begin{abstract}
In this paper, an icosahedral non - body centered model to simulate the periodic structure of homogeneous particulate composites, by predicting the particle arrangement, is presented. This model has yielded three different variations which correspond at three different deterministic particle configurations. In addition, the concept of boundary interphase between matrix and inclusions was taken into account. Thus, the influence of particle vicinity was examined in parallel with the interphase concept on the thermomechanical properties of the overall material.

Next, by the use of this model the authors derived a closed - form expression to estimate the thermal conductivity of this type of composites. To test the validity of the model, the theoretical values arising from the proposed formula were compared with other theoretical predictions obtained from several accurate formulae found in the literature and an adequate accordance was observed.
\end{abstract}

\section{Keywords}

particulate composites, periodic structure, particle contiguity, interphase, thermal conductivity,

\section{Introduction}

It is known that thermal conductivity constitutes a fundamental property of solids and the attainment of its theoretical predictions especially for composites (periodic or not) is a very difficult task, as the thermal conducting mechanism mainly depends on their microstructure.

In the meanwhile, the prediction of the thermomechanical properties of a composite material (fibrous or particulate) is evidently an interesting topic since given that its properties depend on several parameters, such as the individual properties of constituents, the filler size and volume concentration, the adhesion efficiency between inclusions and matrix, the filler distribution and possible vicinity etc.

In this framework, Hashin [1,2] assumed that a particulate composite material is a collection of small volume elements of various sizes and shapes which densely fill the composite. Thus, the particulates were assumed to be conglomerations of spherical inclusions and shells, with the properties of the matrix, surrounding the inclusions. In each volume element, the content of the inclusion was equal to the total content of the dispersed phase in the composite. In addition, Sideridis et al [3] examined the effect of the adhesion between particles and matrix to determine thermal expansion coefficient by a theoretical analysis using a modified model, which includes a third phase, between filler and matrix (the interphase), that has different thermomechanical properties from those of the main phases. On the other hand, Maxwell [4] performed a general basis for estimating the effective thermal conductivity of particulate composites, whereas in Refs. [5-7] some remarkable theoretical and empirical approaches were presented to analyze the thermal conductivity of composites. Further, in Ref. [8] three models on the effective thermal conductivity of concentrated particulate composites were developed via the differential effective medium approach. The first one expresses the relative thermal conductivity of a particulate composite in terms of the thermal conductivity ratio i.e. ratio of dispersed-phase to continuous-phase conductivities and the filler content. The other two models define this property as a function of the above two variables along with the maximum packing volume fraction of particles. Besides, in Ref. [9], the particle contiguity was taken into account to estimate the thermal expansion coefficient for pore free homogeneous composite materials of particle structure while Roudini et al [10] also examined the influence of reinforcement contiguity on the thermal expansion of composites reinforced with alumina particles. In addition, for a detailed investigation on the thermal conductivity and diffusivity of a multi - scale concrete composites by means of an effective medium theory one may refer to Garboczi et al [11], while in Ref. [12] a considerable work to study the effect of an inhomogeneous interphase zone on the bulk modulus and conductivity of particulate composites containing spherical inclusions was performed. Moreover, in Ref. [13] the thermal conductivity of periodic particulate composites was evaluated by a body centered cubic model transformed into a hexaphase spherical model. This model, took into account the influence of 
internal and neighboring particles, but neglected the interphase concept. On the other hand, the effective thermal conductivity of a composite sphere in a continuum medium with conduct resistance was studied in Ref. [14], whereas in Ref. [15] thermal resistance - based bounds for the effective conductivity of composites were determined. Also, Agari and Uno [16, 17] proposed a practical and useful formula to calculate thermal conductivity of particulate composites whilst Khan and Muliana [18] proposed a microstructural model to evaluate thermal conductivity and thermal expansion coefficient for particulate composites, taking into account the particle interaction,. Also, in Ref. [19] the thermal conductivity of homogeneous particulate composites of periodic microstructure was estimated by means of a non body centered model which yielded three variations: simple cubic model, side centered cubic model and face centered cubic model. This model took into consideration the particle distribution and contiguity together with the concept of interphase. Moreover, in Ref. [20] a more advanced polyhedral model was introduced to simulate the paricle arrangement and then to evaluate the thermal conductivity of periodic particulates. In the present work, we examine a periodic particulate composite in the frame of an icosahedral non body centered model yielding three different variations. Besides, the inclusions are encircled by an inhomogeneous interphase, the volume fraction of which is generally determined by heat capacity measurements. Next, the corresponding unit cells arising from these distinct variations are transformed in a unified manner into a nine - phase spherical unit cell. This topological transformation is based on the equality of volume fractions between the corresponding phases. In this context, via this advanced multiphase model, the contiguity amongst the particles, in the form of three deterministic configurations, is examined in parallel with the interphase concept, to estimate the thermal conductivity of the overall material by a modified form of Agari and Uno model [16].

\section{Simulation of particle arrangement}

The majority of microstructural models aim at reproducing in space the basic cell or Representative Volume Element (RVE) of the periodic composite at a microscopic scale in order to obtain a solution. Such models are usually based on the following assumptions:

1) A regular geometric form is generally adopted for the inclusions usually a sphere.

2) Regular geometry and topology are applied for such simulations. Models can be plane or spatial. It is obvious that a three dimensional structure is synonym to the overall material periodic microstructure.

Now, the first variation of the proposed icosahedral model of edge $\mathbf{L}$ appearing in Fig. 1 constitutes an advanced three dimensional system capable of simulating real particulate composites.

By focusing on this model, it can be observed that 30 inclusions occupy the midpoints of all edges whereas 12 inclusions occupy the vertices of this regular polyhedron.

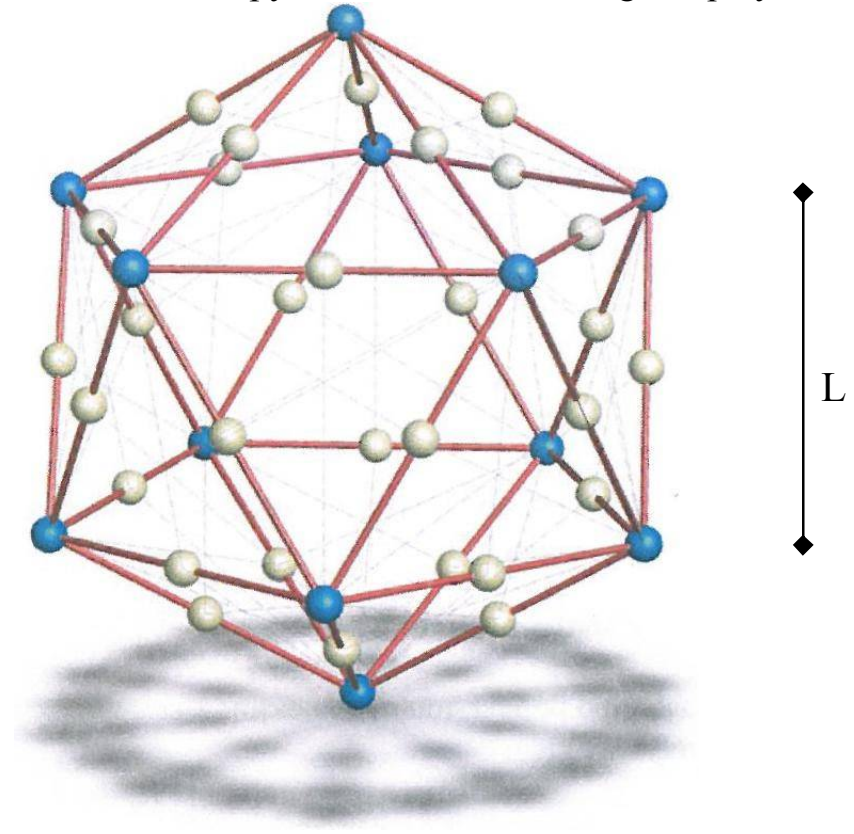

Fig. 1 First variation of the icosahedral model

It is known from Euclidean Geometry that the radii of the unique circumscribed and inscribed sphere respectively are given as 
$R_{20}=\frac{L}{2} \sqrt{\frac{5+\sqrt{5}}{2}} ; r_{20}=L \sqrt{3} \cdot \frac{(3+\sqrt{5})}{12}$

and therefore

$r_{20}=R_{20} \cdot \sqrt{\frac{5+2 \sqrt{5}}{15}}$

Moreover, since a regular icosahedron consists of 20 equilateral triangles that are obviously equal to each other, the surface area is given as

$A_{20}=20 \frac{L^{2} \sqrt{3}}{4}=5 \sqrt{3} L^{2}$

or equivalently

$A_{20}=(10 \sqrt{3}-2 \sqrt{15}) R_{20}^{2}$

Besides the volume is given as

$V_{20}=\frac{1}{3} A_{20} \cdot r_{20}=\frac{5(3+\sqrt{5})}{12} L^{3}$

or equivalently

$V_{20}=\frac{2 \sqrt{10+2 \sqrt{5})}}{3} R^{3}$

Next, the unit cell or RVE which corresponds at the above model in a unique manner may be defined as a regular icosahedron of edge $2 L$, which surrounds the model of Fig. 1 having the same centroid and is reproduced in space in a symmetrical manner to describe a periodic particulate composite.

Then to facilitate the mathematical analysis, utilizing the evident structural symmetries, one may transform this aforementioned unit cell into a 5 - phase spherical model consisting of five concentric spheres of radii a, b, c, d, e respectively such that $\mathrm{a}<\mathrm{b}<\mathrm{c}<\mathrm{d}<\mathrm{e}$

The cross - sectional area of this model is illustrated in Fig. 2

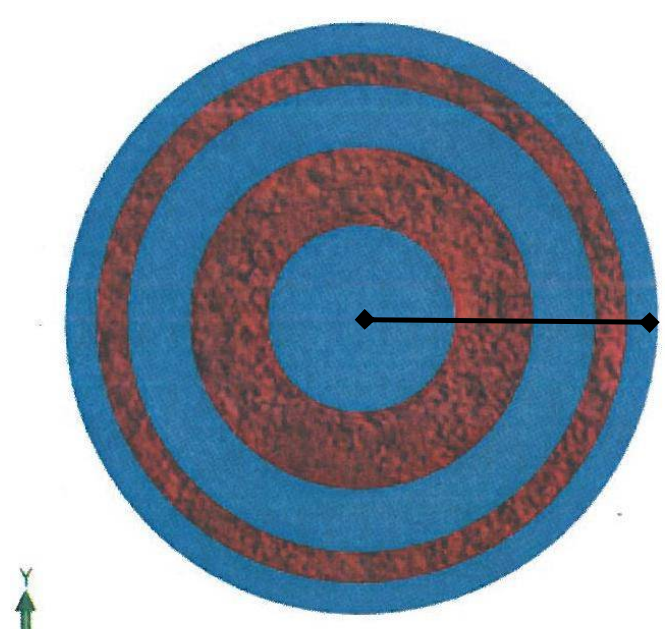

a; b; c; d; e

Fig. 2 Five - phase spherical model

The structure of this multiphase unit cell, which does not contain any interphase zone, is described as follows:

The first and third and fifth phase, (the spherical region of inner radius a, and outer radius $b$, the sector of inner radius $b$ and outer radius $c$ and the zone of inner radius $d$ and outer radius $e)$, represent the matrix.

In addition, the second and forth layer represent the filler. In the meanwhile, the volume of the above mentioned RVE i.e. the circumscribed icosahedron of edge $2 L$ is given as 
$\mathrm{V}_{\mathrm{RVE}}=\frac{5(3+\sqrt{5})}{12} \cdot(2 L)^{3}$

Next, by assuming equal inclusions, which is not necessarily the case in reality, the volume fraction, $U_{\mathrm{f}}$, of the fillers in the continuous matrix is given in terms of the filler radius as folows

$$
\mathrm{U}_{\mathrm{f}}=\frac{42 \frac{4}{3} \pi \cdot \mathrm{r}_{\mathrm{f}}{ }^{3}}{\frac{5(3+\sqrt{5})}{12} \cdot(2 L)^{3}}
$$

with $r_{\mathrm{f}}$ denotes the filler radius which obviously is considered as known.

The above relationship can be solved for $L$ to yield

$$
L=\sqrt[3]{\frac{84 \pi \cdot \mathrm{r}_{\mathrm{f}}^{3}}{5(3+\sqrt{5}) \mathrm{U}_{\mathrm{f}}}}
$$

According to the proposed topological transformation, the volume of the icosahedral unit cell of edge $2 L$ reduces to the volume of a sphere of outer radius $e$. Hence one infers

$$
(2 L)^{3}=\frac{4}{3} \pi e^{3}
$$

Eqn. (6) can be combined with eqns. (5a) and (5b) to yield

$$
e=\mathrm{r}_{\mathrm{f}} \sqrt[3]{\frac{42}{\mathrm{U}_{\mathrm{f}}}}
$$

Now let us denote as $\mathrm{w}_{20}$ the midradius of the icoahedral model of Fig. 1, i.e. the distance from the centroid to the midpoint of an arbitrary edge.

From Euclidean Geometry it is known that

$$
\mathrm{w}_{20}=\frac{1}{4}(1+\sqrt{5}) L
$$

Here, we may assume without violating the rigor of our mathematical formalism that the spherical sectors in the multiphase cell of Fig. 2 expressing the two groups of particles in Fig. 1 (i.e. 30 and 12) are equidistantly distributed on both sides of the spherical surfaces defined by radii $\mathrm{w}_{20}$ and $\mathrm{R}_{20}$

Hence, these phases are developed in such a way that to be in accordance with the following equalities:

$$
\begin{aligned}
& b^{3}-a^{3}=30 r_{f}^{3} \\
& b^{3}+a^{3}=2 w_{20}^{3}
\end{aligned}
$$

and

$\mathrm{d}^{3}-\mathrm{c}^{3}=12 \mathrm{r}_{\mathrm{f}}^{3}$

$\mathrm{d}^{3}+\mathrm{c}^{3}=2 \mathrm{R}_{20}^{3}$

The solution of the above groups of equations yields the values of a,b,c,d in terms of filler radius as follows:

$$
\mathrm{a}=\sqrt[3]{\mathrm{w}_{20}^{3}-15 \mathrm{r}_{\mathrm{f}}^{3}} ; \mathrm{b}=\sqrt[3]{\mathrm{w}_{20}^{3}+15 \mathrm{r}_{\mathrm{f}}^{3}} ; \mathrm{c}=\sqrt[3]{\mathrm{R}_{20}^{3}-6 \mathrm{r}_{\mathrm{f}}^{3}} ; \mathrm{d}=\sqrt[3]{\mathrm{R}_{20}^{3}+6 \mathrm{r}_{\mathrm{f}}^{3}}
$$

Moreover, the following geometric restrictions hold 
i) $\mathrm{a}>0 \Rightarrow \mathrm{a}^{3}>0 \Leftrightarrow \mathrm{w}_{20}^{3}-15 \mathrm{r}_{\mathrm{f}}^{3}>0$

Inequality (12) by the aid of eqns. (5b) and (8) yields

$\left(\frac{1}{4}(1+\sqrt{5})\right)^{3} \frac{84 \pi \cdot r_{f}{ }^{3}}{5(3+\sqrt{5}) U_{f}}-15 r_{f}^{3}>0$

Solving for $\mathrm{U}_{\mathrm{f}}$ one finds

$\mathrm{U}_{\mathrm{f}}<0.355824$

ii) $c>0 \Rightarrow c^{3}>0 \Leftrightarrow R_{20}^{3}-6 r_{f}^{3}>0$

Inequality (14) by the aid of eqns. (1a) and (5b) yields

$$
\frac{84 \pi \cdot \mathrm{r}_{\mathrm{f}}{ }^{3}}{5(3+\sqrt{5}) \mathrm{U}_{\mathrm{f}}}\left(\frac{1}{2} \sqrt{\frac{5+\sqrt{5}}{2}}\right)^{3}-6 \mathrm{r}_{\mathrm{f}}^{3}>0
$$

Solving for $\mathrm{U}_{\mathrm{f}}$ one finds

$\mathrm{U}_{\mathrm{f}}<1.44$

Here, one may observe that the above inequality holds identically and thus it does not constitute a constraint for the first variation of the proposed icosahedral model.

iii) b $<\mathrm{c} \Rightarrow \mathrm{b}^{3}<\mathrm{c}^{3} \Leftrightarrow$

$\left(\frac{1}{4}(1+\sqrt{5})\right)^{3} \frac{84 \pi \cdot \mathrm{r}_{\mathrm{f}}{ }^{3}}{5(3+\sqrt{5}) \mathrm{U}_{\mathrm{f}}}+15 \mathrm{r}_{\mathrm{f}}^{3}<\frac{84 \pi \cdot \mathrm{r}_{\mathrm{f}}{ }^{3}}{5(3+\sqrt{5}) \mathrm{U}_{\mathrm{f}}}\left(\frac{1}{2} \sqrt{\frac{5+\sqrt{5}}{2}}\right)^{3}-6 \mathrm{r}_{\mathrm{f}}^{3}$

Solving for $\mathrm{U}_{\mathrm{f}}$ one finds

$\mathrm{U}_{\mathrm{f}}<0.158748$

iii) $\mathrm{d}<\mathrm{e} \Rightarrow \mathrm{d}^{3}<\mathrm{e}^{3} \Leftrightarrow \mathrm{R}_{20}^{3}+6 \mathrm{r}_{\mathrm{f}}^{3}<\mathrm{r}_{\mathrm{f}}^{3} \frac{42}{\mathrm{U}_{\mathrm{f}}}$

Here we took into account eqn. (7)

Thus it follows

$\frac{84 \pi \cdot \mathrm{r}_{\mathrm{f}}{ }^{3}}{5(3+\sqrt{5}) \mathrm{U}_{\mathrm{f}}}\left(\frac{1}{2} \sqrt{\frac{5+\sqrt{5}}{2}}\right)^{3}+6 \mathrm{r}_{\mathrm{f}}^{3}<\mathrm{r}_{\mathrm{f}}{ }^{3} \frac{42}{\mathrm{U}_{\mathrm{f}}}$

Solving for $\mathrm{U}_{\mathrm{f}}$ one finds

$\mathrm{U}_{\mathrm{f}}<5.5$

Here, one may deduce that the above constraint holds identically and hence it does not affect the validity of the first variation of the model.

In continuing, let us consider the second variation of the introduced non - body centered icosahedral model [Fig.3] 


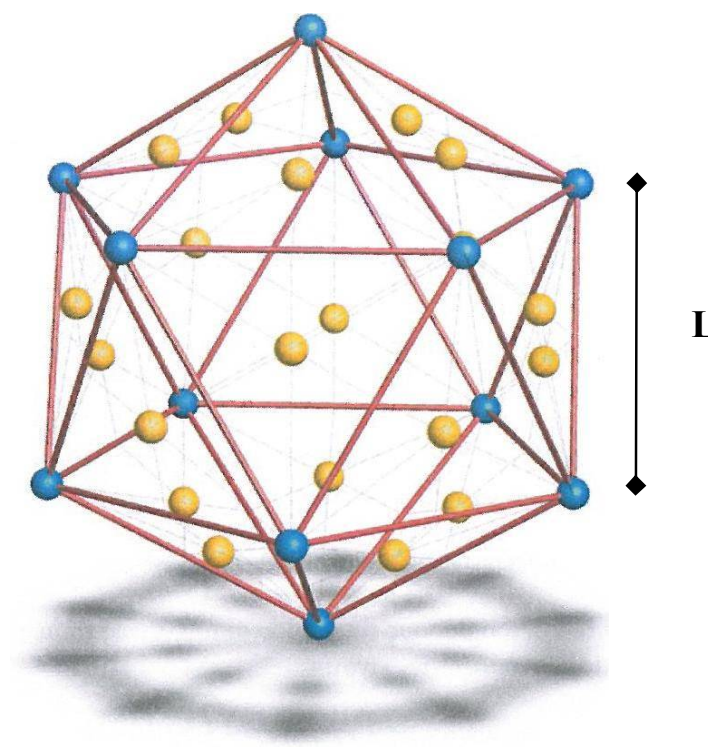

Fig. 3 Second variation of the icosahedral model

By centering on this model, one may perceive that 20 inclusions occupy the centroids of all faces whereas 12 inclusions occupy the vertices of this platonic solid.

Following the same reasoning as before, the RVE which corresponds at the above model in a unique manner may be defined as a regular icosahedron of edge $2 L$, which surrounds the model of Fig. 3 having the same centroid and is reproduced in space in a symmetrical manner to describe the material.

Next, one may point out that this unit cell, after the same topological transformation that we previously carried out, results in the same 5 - phase spherical model of Fig. 2

The volume of the above mentioned RVE i.e. the circumscribed icosahedron of edge $2 L$ is given as

$$
\mathrm{V}_{\mathrm{RVE}}=\frac{5(3+\sqrt{5})}{12} \cdot(2 L)^{3}
$$

Next, by assuming equal inclusions, which is not necessarily the case in reality, the volume fraction $U_{f}$, of the fillers inside the continuous matrix is given in terms of the filler radius as folows

$$
\mathrm{U}_{\mathrm{f}}=\frac{42 \frac{4}{3} \pi \cdot \mathrm{r}_{\mathrm{f}}{ }^{3}}{\frac{5(3+\sqrt{5})}{12} \cdot(2 L)^{3}}
$$

with $r_{f}$ denotes the filler radius which obviously is considered as known.

The above relationship can be solved for $L$ to yield

$$
L=\sqrt[3]{\frac{84 \pi \cdot \mathrm{r}_{\mathrm{f}}{ }^{3}}{5(3+\sqrt{5}) \mathrm{U}_{\mathrm{f}}}}
$$

Taking into account the proposed geometric transformation, the volume of a square polyhedron of edge $2 \ell$ reduces again to the volume of a sphere of radius $e$. Hence we infer

$$
(2 \ell)^{3}=\frac{4}{3} \pi \mathrm{e}^{3} \Rightarrow \mathrm{e}=2 \ell \sqrt{\frac{1}{\pi}}
$$

Eqn. (20) can be combined with eqn. (19) to yield 
$\mathrm{e}=\mathrm{r}_{\mathrm{f}} \sqrt[3]{\frac{32}{U_{\mathrm{f}}}}$

In addition, it is obvious that the first phase of the spherical model [Fig. 3] should surround the centroid axis of the square polyhedron and therefore the validity of eqn. (5) is also extended to the second prismatic model.

Moreover, since we have already considered that in the 4 - phase model occurring in Fig. 3 the circle of radius $w_{20}$, lies in the middle of the circular section denoting the third phase, eqn. (7) still holds.

Hence, in proportion with the first variation one may write out

$\mathrm{b}^{3}-\mathrm{a}^{3}=20 \mathrm{r}_{\mathrm{f}}^{3} ; \mathrm{b}^{3}+\mathrm{a}^{3}=2 \mathrm{w}_{20}^{3}$
$\mathrm{~d}^{3}-\mathrm{c}^{3}=12 \mathrm{r}_{\mathrm{f}}^{3} ; \mathrm{d}^{3}+\mathrm{c}^{3}=2 \mathrm{R}_{20}^{3}$

Thus one infers

$\mathrm{a}=\sqrt[3]{\mathrm{w}_{20}^{3}-10 \mathrm{r}_{\mathrm{f}}^{3}} ; \mathrm{b}=\sqrt[3]{\mathrm{w}_{20}^{3}+10 \mathrm{r}_{\mathrm{f}}^{3}} ; \mathrm{c}=\sqrt[3]{\mathrm{R}_{20}^{3}-6 \mathrm{r}_{\mathrm{f}}^{3}} ; \mathrm{d}=\sqrt[3]{\mathrm{R}_{20}^{3}+6 \mathrm{r}_{\mathrm{f}}^{3}} \quad(24 \mathrm{a}, \mathrm{b}, \mathrm{c}, \mathrm{d})$

Furthermore, the geometric constraints expressed by eqns. (10 a,b) hold for this model as well.

Now, the first restriction yields

$\mathrm{b}^{2}>\mathrm{a}^{2} \Rightarrow \mathrm{w}^{2}+\frac{\mathrm{r}_{\mathrm{f}}^{4}}{\mathrm{w}^{2}}-2 \mathrm{r}_{\mathrm{f}}^{2} \geq \mathrm{r}_{\mathrm{f}}^{2} \Rightarrow$

$\frac{1}{2} \cdot r_{f}^{2} \frac{5 \pi}{4 U_{f}}+\frac{r_{f}^{4}}{\frac{1}{2} \cdot r_{f}^{2} \frac{5 \pi}{4 U_{f}}}-3 r_{f}^{2} \geq 0 \Rightarrow$

$\frac{1}{\frac{1}{2} \cdot \frac{5 \pi}{4}} \cdot \mathrm{U}_{\mathrm{f}}^{2}-3 \mathrm{U}_{\mathrm{f}}+\frac{1}{2} \cdot \frac{5 \pi}{4} \geq 0 \Rightarrow$

$\mathrm{U}_{\mathrm{f}} \leq 0.7499$

Also, according to the second restriction one infers

$\mathrm{d}^{2}>\mathrm{c}^{2} \Rightarrow \mathrm{r}_{\mathrm{f}}{ }^{2} \frac{5}{\mathrm{U}_{\mathrm{f}}} \geq \frac{2}{4} \mathrm{r}_{\mathrm{f}}{ }^{2} \frac{5 \pi}{4 \mathrm{U}_{\mathrm{f}}}+\frac{\mathrm{r}_{\mathrm{f}}^{2}}{\frac{2}{4} \cdot \frac{5 \pi}{4 \mathrm{U}_{\mathrm{f}}}}+2 \mathrm{r}_{\mathrm{f}}{ }^{2} \Rightarrow \frac{\mathrm{U}_{\mathrm{f}}^{2}}{\frac{2}{4} \cdot \frac{5 \pi}{4}}+2 \mathrm{U}_{\mathrm{f}}+\left(\frac{2}{4} \cdot \frac{5 \pi}{4}-5\right) \leq 0 \Rightarrow$

$\mathrm{U}_{\mathrm{f}} \leq 1.1698$

Finally, let us present the third variation [Fig.4]

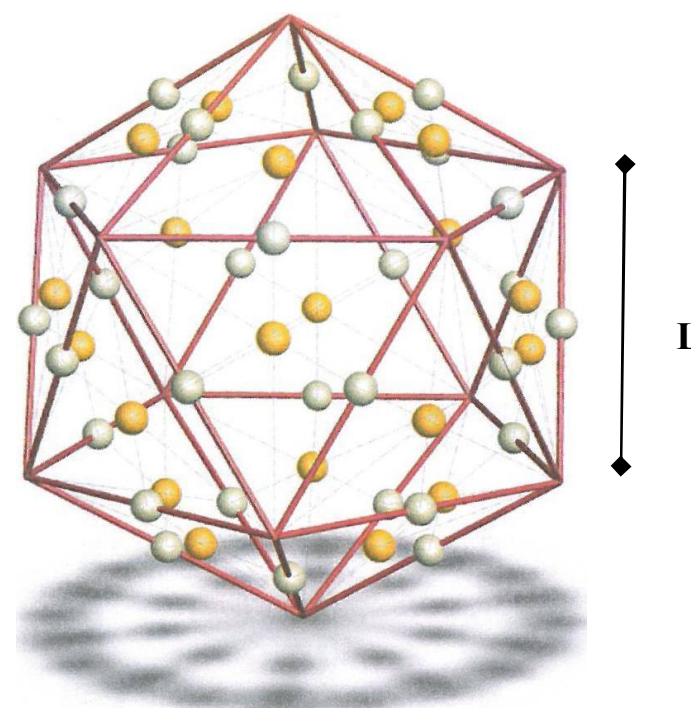

$\mathbf{L}$

Fig. 4 Third variation of icosahedral model 
Here, one may pinpoint that 20 inclusions occupy the midpoints of the edges.

By proceeding as before one may write out

$$
\mathrm{U}_{\mathrm{f}}=\frac{50 \frac{4}{3} \pi \cdot \mathrm{r}_{\mathrm{f}}{ }^{3}}{\frac{5(3+\sqrt{5})}{12} \cdot(2 L)^{3}}
$$

and therefore

$$
L^{3}=\frac{20 \pi \cdot \mathrm{r}_{\mathrm{f}}{ }^{3}}{(3+\sqrt{5}) \mathrm{U}_{\mathrm{f}}}
$$

Hence it follows

$$
(2 \ell)^{3}=\frac{4}{3} \pi \mathrm{e}^{3} \Rightarrow e=r_{\mathrm{f}} \sqrt[3]{\frac{50 \pi}{U_{\mathrm{f}}}}
$$

In analogy to the previously examined variations of the non body centered icosahedral model the following relationships hold

$$
\begin{aligned}
& b^{3}-a^{3}=20 r_{f}^{3} ; b^{3}-a^{3}=2 w_{20}^{3}(30 a, b) \\
& d^{3}-c^{3}=30 r_{f}^{3} ; d^{3}-c^{3}=2 R_{20}^{3}(31 a, b)
\end{aligned}
$$

and therefore

$$
\mathrm{a}=\sqrt[3]{\mathrm{w}_{20}^{3}-10 \mathrm{r}_{\mathrm{f}}^{3}} ; \mathrm{b}=\sqrt[3]{\mathrm{w}_{20}^{3}+10 \mathrm{r}_{\mathrm{f}}^{3}} ; \mathrm{c}=\sqrt[3]{\mathrm{R}_{20}^{3}-15 \mathrm{r}_{\mathrm{f}}^{3}} ; \mathrm{d}=\sqrt[3]{\mathrm{R}_{20}^{3}+15 \mathrm{r}_{\mathrm{f}}^{3}}
$$

Moreover, according to the same reasoning as before one may set the following constraints concerning filler concentration. In this framework, one may write out

$\mathrm{a}>0 \Leftrightarrow \mathrm{w}_{20}^{3}-10 \mathrm{r}_{\mathrm{f}}^{3}>0$

After the necessary algebraic manipulations one obtains

$U_{\mathrm{f}}<0.517998$

Next, one may observe that the following inequality holds

$c>0 \Leftrightarrow \mathrm{R}_{20}^{3}-15 \mathrm{r}_{\mathrm{f}}^{3}>0$

and therefore

$U_{\mathrm{f}}<0.4236$

In continuing, it is evident that the following inequality holds

$$
b<\mathrm{c} \Rightarrow b^{3}<\mathrm{c}^{3} \Leftrightarrow \mathrm{w}_{20}^{3}-10 \mathrm{r}_{\mathrm{f}}^{3}<\mathrm{R}_{20}^{3}-15 \mathrm{r}_{\mathrm{f}}^{3}
$$

After some algebra one finds

$U_{\mathrm{f}}<0.04696$

Finally, it is obvious that the following inequality holds 
$d<\mathrm{e} \Rightarrow d^{3}<\mathrm{e}^{3} \Leftrightarrow \mathrm{R}_{20}^{3}+15 \mathrm{r}_{\mathrm{f}}^{3}<\frac{50}{U_{\mathrm{f}}} \mathrm{r}_{\mathrm{f}}^{3}$

After the necessary algebraic manipulations one obtains

$$
U_{\mathrm{f}}<2.9
$$

Thus one may deduce that the third variation of the icosahedral model is valid at $U_{\mathrm{f}}<0.04696$ and thus one concludes that this variation is rather impractical. For facility reasons let us summarize in Fig.7 the unified topological transformation of the three variations of the non body unit cells. Note that the three icosahedra below, are surrounded by matrix, which corresponds at the outer zone of the multilayer spherical model.

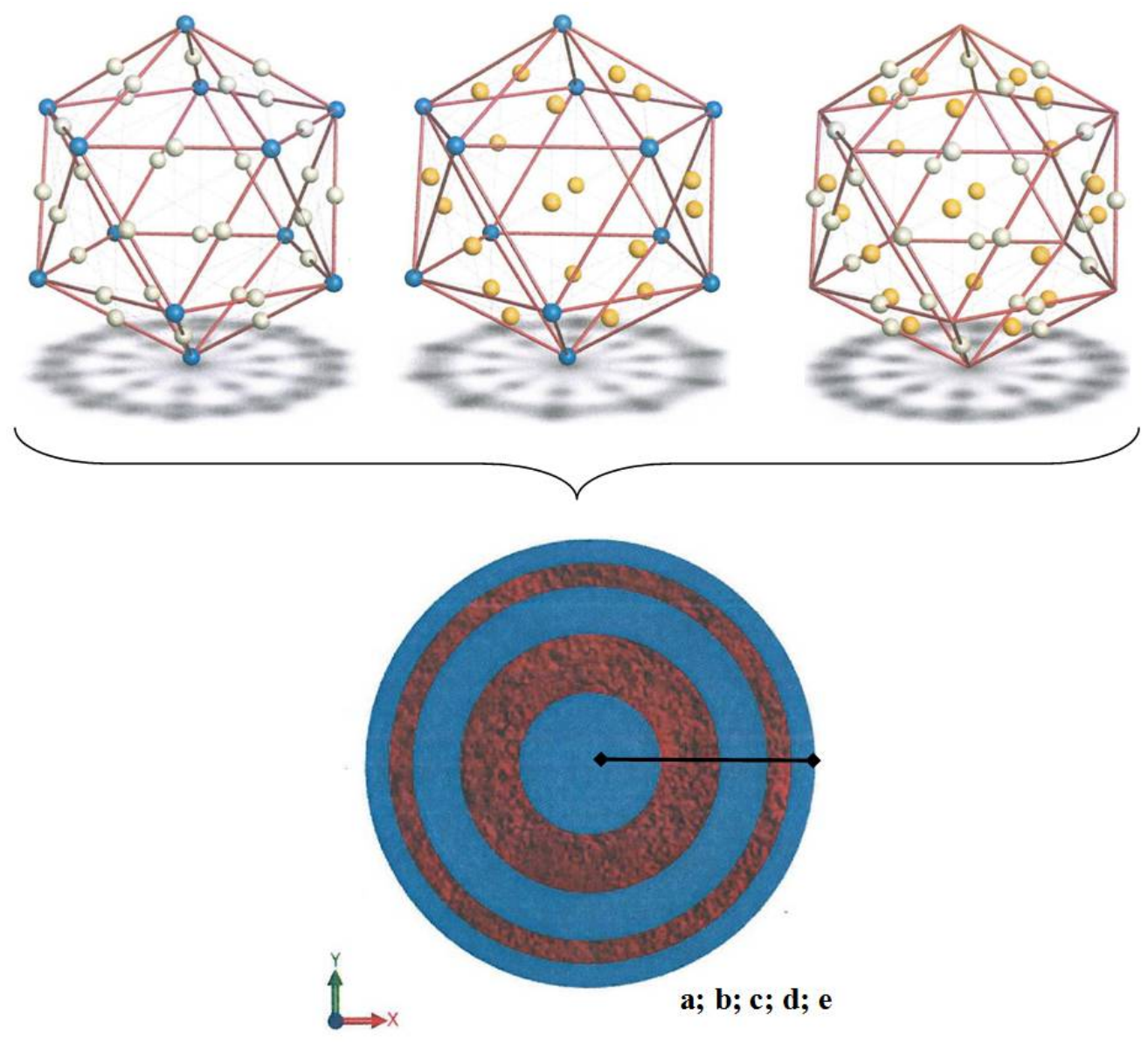

Fig. 5 Transformation of all phases

\section{The concept of interphase - Towards a 9 - phase spherical model}

As we have mentioned before initiating our previous analysis, we are going to simulate the periodic microstructure of a homogeneous particulate composite material by means of unit cells based on icosahedral body centered models, when the inclusions are also encircled by an inhomogeneous interphase region. In this context, in order to perform a more refined analysis, according to the same reasoning as before, the three basic icosahedral body centered models of Fig. $(1,3,4)$, which are enhanced now by the existence of an inhomogeneous interphase zone around the particles, are transformed into an nine - phase spherical model.

Evidently, this multiphase model can be interpreted in the sense of the nine-phase model of Fig. 8 since the interphase regions are developed at the interfaces between filler and matrix. 

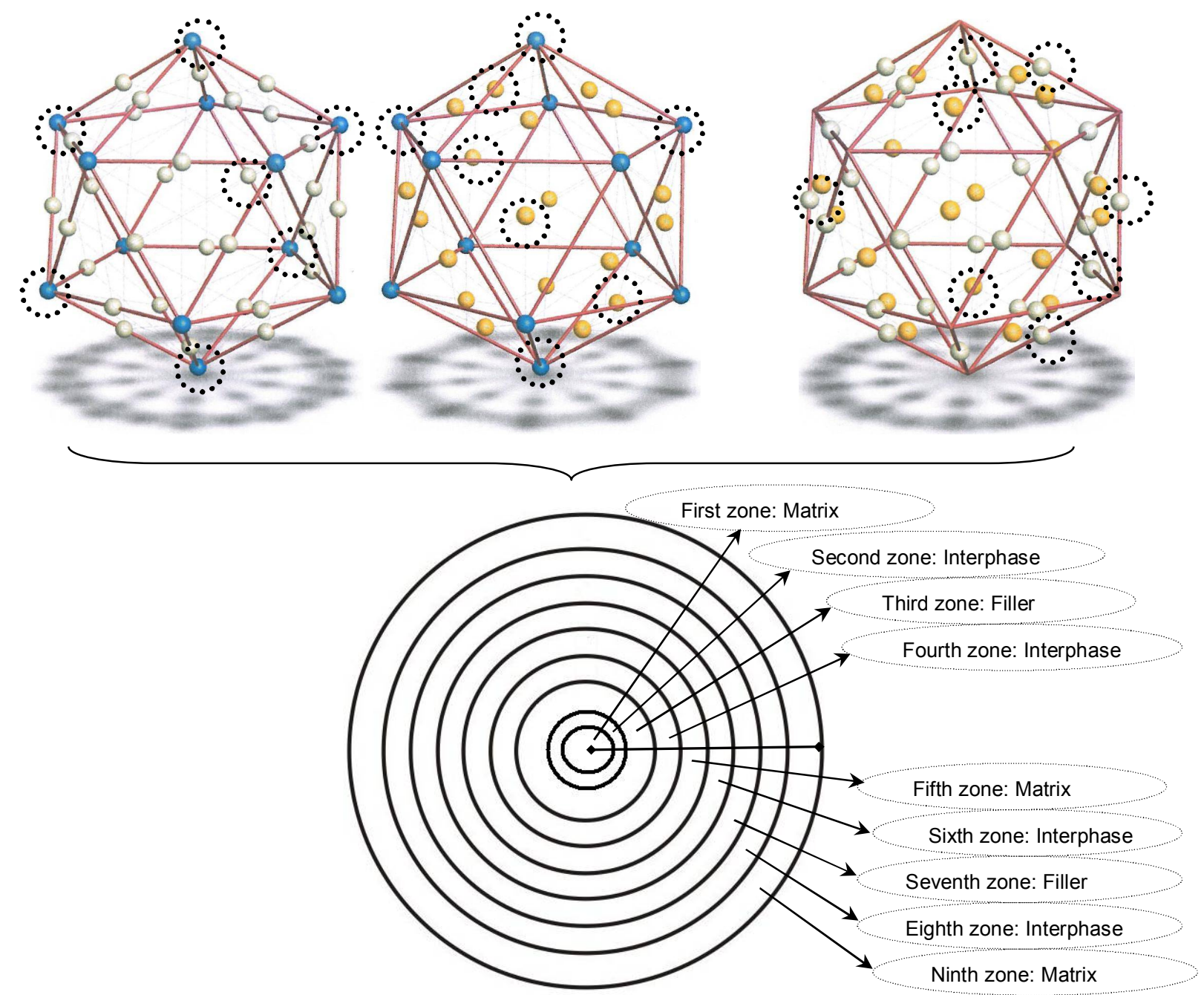

$\mathrm{r}_{1} ; \mathrm{r}_{2} ; \mathrm{r}_{3} ; \mathrm{r}_{4} ; \mathrm{r}_{5} ; \mathrm{r}_{6} ; \mathrm{r}_{7} ; \mathrm{r}_{8} ; \mathrm{r}_{9}$

Fig. 6 Transformation of the new unit cells into a 9 - phase spherical RVE

Evidently, the following relationships hold

$U_{\mathrm{f}}=U_{3}+U_{7}$

$U_{i}=U_{2}+U_{4}+U_{6}+U_{8}$

$U_{\mathrm{m}}=U_{1}+U_{5}+U_{9}$

Besides, since that the interphase is considered somewhat as an altered matrix and its proportion is constant as developed in the interfaces of the two basic constituents of the composite, one may suppose without violating the generality that

$U_{i, 1}=U_{i, 2} \quad ; U_{i, 3}=U_{i, 4}$

Hence one may write out

$\frac{U_{i, 1}}{U_{m, 1}}=\frac{U_{i, 3}}{U_{m, 3}}=\frac{U_{i}}{U_{m}}=\frac{U_{i}}{1-U_{\mathrm{f}}-U_{i}}=k$ 
In this context, one may evaluate the radii of the interphase layers developed around the concentric spheres of the proposed nine - layer coaxial spherical model by means of the following procedure

$U_{2}=k U_{1} \Leftrightarrow \frac{\frac{4}{3} \pi\left(r_{2}^{3}-r_{1}^{3}\right)}{\frac{4}{3} \pi r_{9}^{3}}=k \frac{\frac{4}{3} \pi\left(r_{1}^{3}\right)}{\frac{4}{3} \pi r_{9}^{3}} \Leftrightarrow r_{2}^{3}-r_{1}^{3}=k r_{1}^{3} \Leftrightarrow$

$r_{2}=r_{1} \sqrt[3]{k+1}$

$U_{4}=k U_{5} \Leftrightarrow \frac{\frac{4}{3} \pi\left(r_{4}^{3}-r_{3}^{3}\right)}{\frac{4}{3} \pi r_{9}^{3}}=k \frac{\frac{4}{3} \pi\left(r_{5}^{3}-r_{4}^{3}\right)}{\frac{4}{3} \pi r_{9}^{3}} \Leftrightarrow r_{4}^{3}-r_{3}^{3}=k\left(r_{5}^{3}-r_{4}^{3}\right) \Leftrightarrow$

$r_{4}=\sqrt[3]{\frac{k r_{5}^{3}+r_{3}^{3}}{k+1}}$

$U_{6}=k U_{5} \Leftrightarrow \frac{\frac{4}{3} \pi\left(r_{6}^{3}-r_{5}^{3}\right)}{\frac{4}{3} \pi r_{9}^{3}}=k \frac{\frac{4}{3} \pi\left(r_{5}^{3}-r_{4}^{3}\right)}{\frac{4}{3} \pi r_{9}^{3}} \Leftrightarrow r_{6}^{3}-r_{5}^{3}=k\left(r_{5}^{3}-r_{4}^{3}\right) \Leftrightarrow$

$r_{6}=\sqrt{(k+1) r_{5}^{3}-k r_{4}^{3}}$

$U_{8}=k U_{9} \Leftrightarrow \frac{\frac{4}{3} \pi\left(r_{8}^{3}-r_{7}^{3}\right)}{\frac{4}{3} \pi r_{9}^{3}}=k \frac{\frac{4}{3} \pi\left(r_{9}^{3}-r_{8}^{3}\right)}{\frac{4}{3} \pi r_{9}^{3}} \Leftrightarrow r_{8}^{3}-r_{7}^{3}=k\left(r_{9}^{3}-r_{8}^{3}\right) \Leftrightarrow$

$r_{8}=\sqrt[3]{\frac{k r_{9}^{3}+r_{7}^{3}}{k+1}}$

In continuing, one may obtain the volume fractions of the nine phases as follows

$U_{1}=\frac{\frac{4}{3} \pi r_{1}^{3}}{\frac{4}{3} \pi r_{9}^{3}}=\frac{r_{1}^{3}}{r_{9}^{3}}$

$U_{2}=\frac{\frac{4}{3} \pi\left(r_{2}^{3}-r_{1}^{3}\right)}{\frac{4}{3} \pi r_{9}^{3}}=\frac{r_{2}^{3}-r_{1}^{3}}{r_{9}^{3}}$ 
$U_{3}=\frac{\frac{4}{3} \pi\left(r_{3}^{3}-r_{2}^{3}\right)}{\frac{4}{3} \pi r_{9}^{3}}=\frac{r_{3}^{3}-r_{2}^{3}}{r_{9}^{3}}$

$U_{4}=\frac{\frac{4}{3} \pi\left(r_{4}^{3}-r_{3}^{3}\right)}{\frac{4}{3} \pi r_{9}^{3}}=\frac{r_{4}^{3}-r_{3}^{3}}{r_{9}^{3}}$

$U_{5}=\frac{\frac{4}{3} \pi\left(r_{5}^{3}-r_{4}^{3}\right)}{\frac{4}{3} \pi r_{9}^{3}}=\frac{r_{5}^{3}-r_{4}^{3}}{r_{9}^{3}}$

$U_{6}=\frac{\frac{4}{3} \pi\left(r_{6}^{3}-r_{5}^{3}\right)}{\frac{4}{3} \pi r_{9}^{3}}=\frac{r_{6}^{3}-r_{5}^{3}}{r_{9}^{3}}$

$U_{7}=\frac{\frac{4}{3} \pi\left(r_{7}^{3}-r_{6}^{3}\right)}{\frac{4}{3} \pi r_{9}^{3}}=\frac{r_{7}^{3}-r_{6}^{3}}{r_{9}^{3}}$

$U_{8}=\frac{\frac{4}{3} \pi\left(r_{8}^{3}-r_{7}^{3}\right)}{\frac{4}{3} \pi r_{9}^{3}}=\frac{r_{8}^{3}-r_{7}^{3}}{r_{9}^{3}}$

$U_{9}=\frac{\frac{4}{3} \pi\left(r_{9}^{3}-r_{8}^{3}\right)}{\frac{4}{3} \pi r_{9}^{3}}=\frac{r_{9}^{3}-r_{8}^{3}}{r_{9}^{3}}$

Hence, the volume fractions of all phases have been expressed in terms of the radii of the concentric embedded spheres which constitute the proposed 9 - phase model.

\section{Estimation of thermal conductivities}

As we have emphasized, the concept of interphase has been considered together with the influence of particle contiguity on the thermomechanical properties of the composite.

Generally, the coefficient of thermal conductivity of this phase $\mathrm{K}_{i}$ can be expressed as an $\mathrm{n}$ - degree polynomial with a single independent variable the radius $r$ [21].

For facility reasons, let us assume a parabolic variation of thermal conductivity with respect to the radius $r$ :

Hence the following equality holds:

$\mathrm{K}_{i}(r)=A r^{2}+B r+C$

with $r_{\mathrm{f}} \leq r \leq r_{i}$

The following boundary conditions hold

$r=r_{\mathrm{f}} \Rightarrow \mathrm{K}_{i}(r)=\eta \mathrm{K}_{\mathrm{f}}$

$r=r_{i} \Rightarrow \mathrm{K}_{i}(r)=\mathrm{K}_{m}(r)$ 
The indicator $\eta$ designates the influence of interphase of the thermomechanical properties of composite and its rates belong to the interval $(0,1]$. Yet, one may suppose the maximum influence of interphase both on the thermal conductivity of this intermediate zone and the overall material by supposing that this indicator equals unity [19, 20]

Now, to estimate the terms $A, B$ and $C$ which depend on the radii as well as the thermal conductivities of constituents of the composite, one may apply the previous boundary conditions and in addition, may suppose that all the parabolas which represent graphically this aforementioned variation should have global minima at the critical values $r_{i}$. The corresponding mathematical expression of this requirement, can be formulated as follows

At $r=r_{i}$

$$
\begin{aligned}
& \frac{d \mathrm{~K}_{i}}{d r}=0 \\
& \frac{d^{2} \mathrm{~K}_{i}}{d r^{2}}>0
\end{aligned}
$$

Therefore, after the necessary algebraic manipulation, the following relationship arises

$\mathrm{K}_{i}(r)=\frac{\left(\eta \cdot \mathrm{K}_{f}-\mathrm{K}_{m}\right)\left(r-2 r_{i}\right) r+\eta \cdot \mathrm{K}_{\mathrm{f}} r_{i}^{2}+\mathrm{K}_{m} \cdot r_{\mathrm{f}}^{2}-2 \eta \cdot \mathrm{K}_{m} r_{\mathrm{f}} r_{i}}{\left(r_{i}-r_{\mathrm{f}}\right)^{2}}$

Next, by taking into consideration a rephrased form Agari - Uno formula [16], one infers

$$
\log \mathrm{K}_{c}=\left(U_{1}+U_{4}+U_{7}\right) C_{2} \log \mathrm{K}_{f}+\left(U_{3}+U_{6}+U_{9}\right) \log \left(C_{1} \mathrm{~K}_{m}\right)+\left(U_{2}+U_{5}+U_{8}\right) \log \left(C_{1} \frac{\mathrm{K}_{i, 1}(r)+\mathrm{K}_{i, 2}(r)+\mathrm{K}_{i, 3}(r)}{3}\right)
$$

Here, $C_{1}$ denotes the effect of particles on the secondary structure of the composite whilst $C_{2}$ is a constant related to when the particles begin to form conductive chains. This parameter is introduced in order to modify the assumption that both the matrix phase and particles are all continuous. Evidently the more easily particles are concentrated to shape conductive chains, the more the thermal conductivity of the particles contributes to the variation of the overall the thermal conductivity of system. Here, the authors took into consideration that the interphase is an altered matrix and thus the coefficient $C_{1}$ concerns both matrix and interphase. For facility reasons coefficient $C_{2}$ can be considered equal to unity without violating the generality, wheras $C_{1}$ can be roughly estimated by considering a modified form of particle packing factor defined first in Ref. [21]. In particular, let us focus on the second variation of the icosahedral model given that it has the greatest range of validity when compared with two others. Since the edge of the proposed icosahedron is $L$ it is known that the edge of its unique dual regular dodecahedron defined by the centroids of the icosahedron faces is $\frac{1+\sqrt{5}}{6} L$. Now, let us define the particle packing factor $m$ of the second variation as the ratio between the volumes of icosahedron and the volume of its dual dodecahedron. It is known from the literature [22] that $V_{20}=\frac{5(3+\sqrt{5})}{12} L^{3}$ and $V_{12}=\frac{(65+29 \sqrt{5})}{108} L^{3}$ Hence, one may reasonably conjecture that

$$
C_{1}=\frac{V_{20}}{V_{12}}=\frac{90}{25+11 \sqrt{5}}=\frac{18}{5+\frac{11}{\sqrt{5}}}
$$

To yield the thermal conductivity of the overall material, which as we have already said can be considered as homogeneous, eqn. (63) can generally be combined with (48 - 56) provided of course an experimental estimation of the interphase thickness.

Also, focusing on eqn. (62), one may consider the maximum influence of interphase both on the thermal conductivity of this intermediate zone and the overall material by supposing that $\eta=1$. 
In addition, the average values of interphase conductivities for the two degree parabolic variation law, expressed by eqn. (62) for $\eta=1$ can be estimated as

$$
\begin{aligned}
& \bar{K}_{i, 1}=\frac{2 U_{\mathrm{m}, 1}}{\mathrm{r}_{\mathrm{m}, 1}^{2} \cdot U_{i, 1}} \int_{r_{m, 1}}^{r_{i, 1}} K_{i, 1}(r) d r \\
& \bar{K}_{i, 2}=\frac{2 U_{\mathrm{f}, 1}}{\mathrm{r}_{\mathrm{f}, 1}^{2} \cdot U_{i, 2}} \int_{r_{\mathrm{f}, 1}}^{r_{i, 2}} K_{i, 2}(r) d r \\
& \bar{K}_{i, 3}=\frac{2 U_{\mathrm{m}, 2}}{\mathrm{r}_{\mathrm{m}, 2}^{2} U_{i, 3}} \int_{r_{\mathrm{m}, 2}}^{r_{i, 3}} K_{i, 3}(r) d r \\
& \bar{K}_{i, 4}=\frac{2 U_{\mathrm{f}, 2}}{\mathrm{r}_{\mathrm{f}, 2}^{2} U_{i, 4}} \int_{r_{\mathrm{f}, 2}}^{r_{i, 4}} K_{i, 4}(r) d r
\end{aligned}
$$

\section{Experimental Work}

To define experimentally the interphase thickness, the specimens used have as matrix a system based on a diglycidilether of bisphenol A resin (Epikote 828) as prepolymer, with an epoxy equivalent 185-192, a molecular weight between 370 and 384 and a viscosity of $15000 \mathrm{CP}$ at $25^{\circ} \mathrm{C}$. As curing agent, $8 \%$ triethylenetetramine hardener per weight of the epoxy resin was employed. As filler, aluminum particles of diameter $150 \times 10^{-6} \mathrm{~m}$ and filler contents from $U_{\mathrm{f}}=0.05$ to 0.30 were used.

The properties of the constituent materials are given in Table 1.

\begin{tabular}{|l|l|l|}
\hline Type of constituent material & Aluminum & Epoxy resin \\
\hline Thermal Conductivity & $120 \mathrm{~W} / \mathrm{m} \cdot \mathrm{K}$ & $0.2 \mathrm{~W} / \mathrm{m} \cdot \mathrm{K}$ \\
\hline Density $\rho(\mathrm{kgr} / \mathrm{m} 3)$ & 2700 & 1190 \\
\hline
\end{tabular}

Table 1 Properties of filler and matrix material

The test pieces were machined from each casting. To measure the interphase thickness a series of pure and aluminum reinforced epoxy resin samples of the same diameter and thickness were manufactured and tested on a $\mathrm{Du}$ - Pont 900 differential thermal analyzer combined with a Du - Pont Differential Scanning Calorimetry (DSC) analyzer at the zone of the glass transition temperature in order to determine the specific heat capacity values for filler volume fraction varying from 0 to 30 percent. It is well-known that Lipatov [23] have shown that, if calorimetric measurements are performed in the neighborhood of the glass transition zone of the composite, energy jumps are observed. These jumps are too sensitive to the amount of filler added to the matrix and can be used to evaluate the boundary layers developed around the inclusions. Apparently, as the filler volume fraction is increased, the proportion of macromolecules characterized by a reduced mobility is also increased. This is equivalent to an augmentation of the interphase content and supports the empirical conclusion presented in Ref. [23], which the extent of the interphase expressed by its thickness $\Delta r$ is the cause of the variation of the amplitudes of heat capacity jumps appearing at the glass transition zones of the matrix material and the composite with various filler - volume fractions. Moreover, the size of heat capacity jumps for unfilled and filled materials is directly related to $\Delta r$ by an empirical relationship given in Ref. [23] This expression defines the thickness $\Delta r$ corresponding to the interphase and is written out below This relationship defines the thickness $\Delta r$ corresponding to the interphase and is written out below

$$
\frac{\left(r_{f}+\Delta r\right)^{3}}{r_{f}^{3}}-1=\frac{\lambda U_{f}}{1-U_{f}}
$$


where

$\lambda=1-\frac{\Delta C_{p}^{f}}{\Delta C_{p}^{0}}$

Here, the numerator and the denominator of the fraction appearing in the right hand side of eqn. (70) denote the sudden changes of the heat capacity for the filled and unfilled polymer respectively. In continuing, the values of the interphase thickness and volume fraction for various filler contents are listed in Tables 2-5. These values were obtained by introducing the experimental values of the sudden change and heat capacity at the transition region for the composite into eqns. $(69-70)$.

\begin{tabular}{|c|c|c|}
\hline $\mathrm{U}_{\mathrm{f}}$ & $\Delta \mathrm{r}_{\mathrm{i}}(\mu \mathrm{m})$ & $\mathrm{U}_{\mathrm{i}}$ \\
\hline 0.05 & 0.3 & 0.0013 \\
\hline 0.10 & 1.06 & 0.004 \\
\hline 0.15 & 2.12 & 0.013 \\
\hline 0.20 & 3.53 & 0.028 \\
\hline 0.25 & 5.0 & 0.050 \\
\hline
\end{tabular}

Table 2 Interphase thickness and volume fraction for various filler contents

\begin{tabular}{|c|c|c|c|c|r|r|r|r|}
\hline $\mathrm{r}_{1}(\mu \mathrm{m})$ & $\mathrm{r}_{2}(\mu \mathrm{m})$ & $\mathrm{r}_{3}(\mu \mathrm{m})$ & $\mathrm{r}_{4}(\mu \mathrm{m})$ & $\mathrm{r}_{5}(\mu \mathrm{m})$ & \multicolumn{1}{|c|}{$\mathrm{r}_{6}(\mu \mathrm{m})$} & \multicolumn{1}{|c|}{$\mathrm{r}_{7}(\mu \mathrm{m})$} & \multicolumn{1}{|c|}{$\mathrm{r}_{8}(\mu \mathrm{m})$} & \multicolumn{1}{|c|}{$\mathrm{r}_{9}(\mu \mathrm{m})$} \\
\hline 277.0035 & 277.13 & 290.9511 & 291.1819 & 407.1626 & 447.8789 & 488.5951 & 508.9533 & 529.3114 \\
\hline 213.8782 & 214.196 & 236.0859 & 236.632 & 323.1652 & 355.4817 & 387.7982 & 403.9565 & 420.1148 \\
\hline 181.3065 & 182.2403 & 210.5572 & 212.0603 & 282.3108 & 310.5419 & 338.773 & 352.8885 & 367.004 \\
\hline 159.3733 & 161.2773 & 195.0717 & 197.9276 & 256.4964 & 282.146 & 307.7957 & 320.6205 & 333.4453 \\
\hline 142.6199 & 145.9378 & 184.4561 & 189.0586 & 238.1102 & 261.9212 & 285.7322 & 297.6378 & 309.5433 \\
\hline
\end{tabular}

Table 3 Radii of each region for first icosahedral variation

\begin{tabular}{|c|c|c|c|c|c|c|c|c|}
\hline $\mathrm{r}_{1}(\mu \mathrm{m})$ & $\mathrm{r}_{2}(\mu \mathrm{m})$ & $\mathrm{r}_{3}(\mu \mathrm{m})$ & $\mathrm{r}_{4}(\mu \mathrm{m})$ & $\mathrm{r}_{5}(\mu \mathrm{m})$ & $\mathrm{r}_{6}(\mu \mathrm{m})$ & $\mathrm{r}_{7}(\mu \mathrm{m})$ & $\mathrm{r}_{8}(\mu \mathrm{m})$ & $\mathrm{r}_{9}(\mu \mathrm{m})$ \\
\hline 252.9995 & 253.115 & 277.0119 & 277.4866 & 466.0849 & 512.6934 & 559.3019 & 582.6061 & 605.9104 \\
\hline 189.7454 & 190.0273 & 228.2665 & 229.3624 & 369.9318 & 406.925 & 443.9182 & 462.4148 & 480.9113 \\
\hline 154.815 & 155.6124 & 206.2451 & 209.1962 & 323.1652 & 355.4817 & 387.7982 & 403.9565 & 420.1148 \\
\hline 129.0684 & 130.6103 & 193.2105 & 198.7092 & 293.6151 & 322.9766 & 352.3381 & 367.0189 & 381.6996 \\
\hline 106.6727 & 109.1543 & 184.4599 & 193.1686 & 272.5681 & 299.8249 & 327.0817 & 340.7101 & 354.3385 \\
\hline
\end{tabular}

Table 4 Radii of each region for second icosahedral variation

\begin{tabular}{|c|c|c|c|c|r|r|r|r|}
\hline $\mathrm{r}_{1}(\mu \mathrm{m})$ & $\mathrm{r}_{2}(\mu \mathrm{m})$ & $\mathrm{r}_{3}(\mu \mathrm{m})$ & $\mathrm{r}_{4}(\mu \mathrm{m})$ & $\mathrm{r}_{5}(\mu \mathrm{m})$ & \multicolumn{1}{|c|}{$\mathrm{r}_{6}(\mu \mathrm{m})$} & \multicolumn{1}{|c|}{$\mathrm{r}_{7}(\mu \mathrm{m})$} & \multicolumn{1}{|c|}{$\mathrm{r}_{8}(\mu \mathrm{m})$} & $\mathrm{r}_{9}(\mu \mathrm{m})$ \\
\hline 126.9527 & 127.0106 & 166.0369 & 166.7953 & 369.9318 & 406.925 & 443.9182 & 462.4148 & 480.9113 \\
\hline 73.07607 & 73.18465 & 142.9556 & 144.5607 & 293.6151 & 322.9766 & 352.3381 & 367.0189 & 381.6996 \\
\hline-54.4821 & -54.7627 & 133.3176 & 137.3547 & 256.4964 & 282.146 & 307.7957 & 320.6205 & 333.4453 \\
\hline-75.926 & -76.8331 & 127.9268 & 135.0525 & 233.0424 & 256.3466 & 279.6509 & 291.303 & 302.9551 \\
\hline-84.4967 & -86.4625 & 124.461 & 135.2567 & 216.3374 & 237.9711 & 259.6049 & 270.4218 & 281.2386 \\
\hline
\end{tabular}

Table 5 Radii of each region for third icosahedral variation

\section{Discussion}

In Table 6 , the theoretical values of the thermal conductivity of the overall material with respect to filler volume fraction, as obtained from the authors, eqn. (63), and other workers are performed. Here, we elucidate that the second variation of the icosahedral model was selected for the calculation of thermal conductivity in terms of filler content as it was found to be subjected into the most realistic restrictions for filler concentration by volume. 


\begin{tabular}{|c|c|c|c|c|c|c|c|}
\hline $\mathrm{U}_{\mathrm{f}} \quad 10^{-6} \mathrm{C}^{-1}$ & Authors & Agari and Uno [16] & $\begin{array}{l}\text { Two - Phase Inverse } \\
\text { Law of Mixtures }\end{array}$ & $\begin{array}{l}\text { Three - Phase } \\
\text { Inverse Law of } \\
\text { Mixtures }\end{array}$ & $\begin{array}{l}\text { Kytopoulos } \\
\text { and Sideridis } \\
\text { [13] }\end{array}$ & $\begin{array}{l}\text { Venetis and } \\
\text { Sideridis [19] }\end{array}$ & $\begin{array}{c}\text { Venetis } \\
\text { and } \\
\text { Sideridis } \\
{[20]}\end{array}$ \\
\hline 0 & 0.362 & 0.362 & 0.2 & 0.2 & 0.2 & 0.2 & 0.2 \\
\hline 0.1 & 0.666182 & 0.646779 & 0.222181078 & 0.222403259 & 0.219743998 & 0.24897 & 0.252461 \\
\hline 0.2 & 1.190256 & 1.155588 & 0.249895877 & 0.250145773 & 0.243813239 & 0.279869 & 0.321414 \\
\hline 0.3 & 2.126609 & 2.064669 & 0.28551035 & 0.28779586 & 0.27380382 & 0.315462 & 0.389988 \\
\hline 0.4 & 3.799574 & 3.688906 & 0.332963 & 0.3347893 & 0.31220382 & 0.362884 & 0.494138 \\
\hline
\end{tabular}

Table 6 Theoretical values of the thermal conductivity

In addition, Fig. 7 illustrates the variation of the thermal conductivity of the composite material against filler volume fraction as obtained from the proposed multiphase spherical model, resulting in the modified form of Agari and Uno formula [16]. In addition, theoretical values derived from two and three phase inverse law of mixtures and other formulae also appear [see Appendix Section].

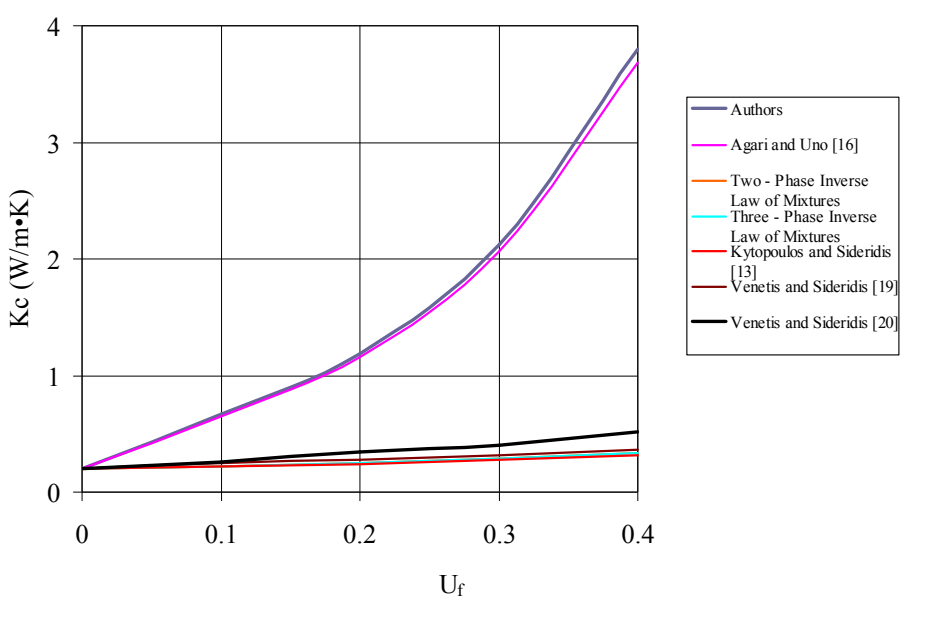

Fig. 7 Theoretical predictions of thermal conductivity versus filler content at low and medium concentrations

Here, one may observe that, as it was expected, at $\mathrm{U}_{\mathrm{f}}=0$ both theoretical values of Agari and Uno formula and eqn. (63) coincide. In addition, for low filler contents, one may point out that eqn. (63) constitutes a slight improvement of Agari and Uno formula; whilst for medium contents up to 0.4 this improvement seems to be more considerable. On the other hand, it is evident that the proposed formula for thermal conductivity yields much better theoretical predictions when compared with inverse mixing laws for two and three phases and the three other theoretical formulae $[13,19,20]$ which were derived on the basis of multiphase forms of inverse law of mixtures given that the application of standard rule of mixtures cannot take place to evaluate this bulk property of a particulate composite. The latter is attributed to the fact that in particulate composites the component phases are interconnected through consecutive spherical phases of filler and matrix [21]. In the meanwhile, one may state that the transformation of a polyhedral model into a multiphase spherical one mainly concerns the implementation of Classical Elasticity approach to particulate composites for the evaluation of properties as tensile modulus, Poisson ratio etc. However, since thermal conductivity is a bulk property of materials one may observe that such a topological transformation is not needed. Yet, thermal conductivity of filled polymers is proved to be analogous to viscosity, tensile modulus, and shear modulus. The following equation demonstrates the numerical relationship between composite material and pure polymer:

$$
\frac{k_{c}}{k_{p}}=\frac{n_{c}}{n_{p}}=\frac{\mathrm{E}_{c}}{\mathrm{E}_{p}}=\frac{G_{c}}{G_{p}}
$$

where the subscripts "c" and "p" denote the composite and pure polymer property respectively. 
Also, in the above equation the symbol " $\mathrm{k}$ " is used to denote thermal conductivity, " $\mathrm{n}$ " to denote viscosity, "E" for the elastic modulus, and "G" for the shear modulus. On the other hand, a shear loading analogy method was proposed by Springer and Tsai [25], to estimate thermal conductivity of a composite. However, this approach mainly concerns fibrous materials whilst the approach of Ref. [24] has indeed a wider validity. Nonetheless, since in many formulae predicting the properties of composites there are limitations referring to the values of filler volume fraction, it is the authors' belief that the aforementioned topological transformation of the icosahedral model into an ninephase spherical one is useful to be carried out towards the estimation of thermal conductivity, since in this way the analogy given by eqn. (71) may be signified and examined in a common framework, when necessary. Moreover, in regard to the possibility of interaction amongst particles which is motivated by the proposed configurations of them, one may say that this is not consistent with the parallel use of inverse law of mixtures. However, in our case this interaction has a qualitative character. Specifically, according to the geometric models for a crystallographic packing of particles in the form of platonic solids, e.g. cubic models, icosahedral models etc., the particle distribution inside the polymer matrix takes place via deterministic configurations. In this way, the range of filler contiguity is defined beforehand in a stringent manner. Hence, given that the development of interphase layers around all inclusions is a fact that cannot be avoided especially in polymer composites filled with inorganic filler; our consideration is in opposition with the undesirable existence of consecutive and/or intersecting - interacting inhomogeneous interphase layers with evidently unspecified thicknesses. In addition, such an unexpected situation may also shift the optimum filler volume fraction above which the reinforcing action of the filler is upset. In our concept, any interphase region is developed solely around each particle and its thickness cannot be affected by the interphase layers of neighboring particles and thus the rule of mixtures can be put into effect as if two "sole interphase layers" to be developed around the "two equivalent neighboring particles", given that according to the two proposed variations of the icosahedral model the particles occupying either the vertices, or the mid - edges, or the mid - faces of the octahedron, constitute three distinct phases as illustrated at the ninephase spherical model. In this context, the thermal conductivity of this category of particulate composites was obtained by exploring the effect of contiguity, something that can be described by the distribution of the particles inside the matrix and which always should be such that to prevent their possible agglomeration.

In addition, one may emphasize that the well known concept of interphase was considered along with the aforementioned influence of particle arrangement, and this combination was illustrated by the performed 9 - phase model, which has merged the influence of these parameters. Thus, one can point out that both these two factors may have an influence on the coefficient of thermal conductivity. Besides, it can be concluded that the simultaneous consideration of these two distinct and important parameters to estimate this property seems quite reasonable since in particulate composites the component phases are interconnected through consecutive spherical phases of filler and matrix.

On the other hand, we should clarify that the proposed model holds for great values of filler content. Furthermore, as a continuation of the present work, the particle configuration could be approached by more advanced deterministic simulations of periodic particulate composites concerning dodecahedral or icosahedral models, divided of course into side centered, face centered and body centered models. Further, the duality of regular polyhedra [22] could be applicable towards the introduction of more advanced representative volume elements of periodic particulate composites. Yet, to predict the particle distribution inside the matrix by the use of symmetrically reproduced unit cells based on platonic solids seems to be a rather simplified approximation when compared with advanced random vector generation techniques, motivated by stochastic methods. However, such approaches despite their inarguable mathematical rigor may not be convenient enough to represent the periodic structure of particle reinforced polymers and incorporate the interphase concept, since they may show many regions of agglomeration, leading to singular and unrealistic results.

\section{Conclusions}

The aim of this study was to develop a microstructural model in order to obtain the thermal conductivity of particulate composites taking into account the contiguity i.e. the existence of several particles in the model (instead of a single particle in the matrix) thus considering the arrangement of the inclusions and its influence on the properties of the composite material. Of course this becomes more important when the filler content of the composite increases and becomes high and/or when the mismatches in the properties of the inclusion and matrix are significant.

The novelty of this work was that the particle contiguity was taken into consideration in parallel with the concept of interphase in order to estimate this property.

To this end, a rigorous mathematical model was performed to simulate particulate composites of periodic structure, containing spherical particles. The material was assumed to be homogeneous and isotropic. 
After all, one may deduce that the interphase magnitude has an effect when the particles are stiffer than the matrix and increases as the thickness does. Meanwhile, the particle configurations indeed have a contribution to the thermal properties for particulate composites with icosahedral arrangements as verified from the transformation of the two possible variations of the proposed icosahedral model, into a nine - phase spherical model.

In closing, it can be said that the theoretical results obtained here may be considered as basic ones for more advanced cell models of this class of composites.

\section{References}

1. Hashin, Z.: The Elastic Moduli of Heterogeneous Materials, J. Appl. Mech. 29, Trans. ASME 84, Series E, pp. 143 -150 (1962)

2. Hashin, Z., Shtrikman, S.: A Variational Approach to the Theory of the Elastic Behavior of Multiphase Materials, J. Mechanics and Physics of Solids 11, pp. 127-140 (1963)

3. E. Sideridis and G. C. Papanicolaou, A theoretical model for the prediction of thermal expansion behaviour of particulate composites Rheologica Acta Vol. 27 pp. 608-616 (1988)

\section{J.C. Maxwell, Electricity and Magnetism. Clarendon, Oxford (1873)}

5. Y. Benveniste, Effective thermal-conductivity of composites with a thermal contact resistance between the constituents-nondilute case. J. Appl. Phys. 61, 2840-2843 (1987)

6. C.W. Nan, R. Birringer, D.R. Clarke, H. Gleiter Effective thermal conductivity of particulate composites with interfacial thermal resistance. J. Appl. Phys. 81, 6692-6699 (1997)

7. H.L. Duan, B.L., Karihaloo, J. Wang, X. Yi, Effective conductivities of heterogeneous media containing multiple inclusions with various spatial distributions. Phys. Rev. B. 73,

174203-174215 (2006)

8. Rajinder Pal New Models for Thermal Conductivity of Particulate Composites Journal of Reinforced Plastics and Composites vol. 26 pp. 7643-651, 2007

9. D.K. Balch, T.J. Fitzgerald, V.J. Michaud, A. Mortensen, Y.-L. Shen, and S. Suresh

Thermal Expansion of Metals Reinforced with Ceramic Particles and Microcellular Foams

Metallurgical and Materials Transactions A

November 1996, Volume 27, Issue 11, pp 3700-3717

10. G. Roudini, R. Tavangar, L. Weber, A. Mortensen: Int. J. Mat. Res. 101 (2010) 1113-1120 Influence of reinforcement contiguity on the thermal expansion of alumina particle reinforced aluminium composites

11. E. J. Garboczi, and J. G. Berryman, New effective medium theory for the diffusivity or conductivity of a multiscale concrete microstructure model, Conc. Sci. and Engin., 2, pp. 88-96, 2000

12. Lutz, M.P. and Zimmerman, R.W. (2005) Effect of an inhomogeneous interphase zone on the bulk modulus and conductivity of a particulate composite, International Journal of Solids and Structures, 42, 429-437.

13. V.N. Kytopoulos E. Sideridis, Thermal Conductivity of Particulate Composites by a Hexaphase Model, JP Journal of Heat and Mass Transfer Volume 13, Number 3, 2016, Pages 395-407

14. J. Felske, Effective thermal conductivity of composite spheres in a continuous medium with contact resistance, Int. J. Heat Mass Trans., Vol. 47, Nos. 14-16, pp.3453-3461, (2004)

15. P. Karayacoubian, M. Yovanovich, and J. Culham, Thermal resistance - based for bounds for the effective conductivity of composite thermal interface materials, 22nd IEEE SEMI - 
THERM Symposium, (2006)

16. Agari Y. J. and Uno T. Jnl. Appl. Pol. Sc. 32, p. 57051986

17. Agari Y. J. and Uno T.Jnl. Appl. Pol. Sc. 43, p. 11171991

18. K. Khan and A. Muliana Effective thermal properties of viscoelastic composites having field-dependent constituent properties. Acta Mech. Vol. 209 pp 153-178, 2010

19. J. Venetis, E. Sideridis, A mathematical model for thermal conductivity of homogeneous composite materials Indian Journal of Pure \& Applied Physics

Vol. 54, May 2016, pp. 313-320

20. Venetis, J. and Sideridis, E. The thermal conductivity of particulate composites by the use of a polyhedral model Colloid Polym Sci (2018) 296: 195. https://doi.org/10.1007/s00396-017-4227-1

21. Theocaris, P. S. The Mesophase Concept in Composites, Polymers/Properties and Applications 11, Springer-Verlag (1987).

22. Peter R. Cromwell Polyhedra Cambridge University Press, 1999

23. Lipatov Y. S.: Physical Chemistry of Filled Polymers, published by Khimiya (Moscow 1977). Translated from the Russian by R. J. Moseley, International Polymer Science and Technology, Monograph No.2

24. D. M. Bigg, Thermally Conductive Polymer Compositions. Polymer Composites, Vol. 7, No. 3, 1986, pp. 125.

25. Springer, G.S, Tsai S. W. Jnl. Comp. Mat., 1, pp. 166-173 (1967)

\section{Appendix}

Let us present the theoretical formulae for thermal conductivity of particulates.

Agari and Uno formula [16]

$\log \mathrm{K}_{c}=U_{f} C_{2} \log \mathrm{K}_{f}+\left(1-U_{f}\right) \log \left(C_{1} \mathrm{~K}_{m}\right)(\mathrm{A} 1)$

The coefficients $C_{1} ; C_{2}$ have already been discussed in the text below eqn. (69) of the current article.

Kytopoulos - Sideridis formula [13]

$$
\frac{1}{\mathrm{~K}_{c}}=\frac{1}{\mathrm{~K}_{m}}+\frac{\left|\mathrm{K}_{m}-\mathrm{K}_{f}\right| U_{f}}{\mathrm{~K}_{f} \mathrm{~K}_{m}} \cdot \max \left\{k_{1}, k_{2}\right\}
$$

Here $k_{1}, k_{2}$ are dimensionless parameters somewhat analogous to the packing factor for periodic particulate composites defined by Theocaris in Ref. [21]. Evidently these parameters lie between 0 and 1.

Venetis - Sideridis formula [19]

$$
\mathrm{K}_{c}=\frac{\mathrm{K}_{m} \mathrm{~K}_{f} \overline{\mathrm{K}}_{i}}{\mathrm{~K}_{f} \overline{\mathrm{K}}_{i}\left(\frac{r_{1}^{3}}{r_{5}^{3}}+\frac{r_{5}^{3}-r_{4}^{3}}{r_{5}^{3}}\right)+\mathrm{K}_{m} \overline{\mathrm{K}}_{i}\left(\frac{r_{3}^{3}-r_{2}^{3}}{r_{5}^{3}}\right)+\mathrm{K}_{f} \mathrm{~K}_{m}\left(\frac{r_{2}^{3}-r_{1}^{3}}{r_{5}^{3}}+\frac{r_{4}^{3}-r_{3}^{3}}{r_{5}^{3}}\right)}
$$


where $r_{1}-r_{5}$ denote the radii of a coaxial five - phase spherical model arising from the transformation of the non body centred RVE

Venetis - Sideridis formula [20]

$$
\mathrm{K}_{c}=\frac{\mathrm{K}_{f} \mathrm{~K}_{m} \overline{\mathrm{K}_{i, c}}}{\mathrm{~K}_{m} \overline{\mathrm{K}_{i, c}}\left(\frac{r_{1}^{2}+r_{5}^{2}-r_{4}^{2}}{r_{7}^{2}}\right)+\mathrm{K}_{f} \overline{\mathrm{K}_{i, c}}\left(1+\frac{r_{3}^{2}-r_{2}^{2}-r_{6}^{2}}{r_{7}^{2}}\right)+\mathrm{K}_{f} \mathrm{~K}_{m}\left(\frac{r_{2}^{2}-r_{1}^{2}+r_{4}^{2}-r_{3}^{2}+r_{6}^{2}-r_{5}^{2}}{r_{7}^{2}}\right)}
$$

Here $r_{1}-r_{7}$ denote the radii of a coaxial seven - phase spherical model arising from the transformation of the body centred RVE. Since the above formula is a rephrased form of inverse mixtures law, the term $\overline{\mathrm{K}_{i, c}}$ is approached by the weighted harmonic mean of $\overline{\mathrm{K}_{i, 1}} ; \overline{\mathrm{K}_{i, 2}} ; \overline{\mathrm{K}_{i, 3}}$ for the three interphase zones and therefore

$$
\overline{\mathrm{K}_{i, c}}=\frac{3}{\frac{1}{\overline{\mathrm{K}_{i, 1}}}+\frac{1}{\overline{\mathrm{K}_{i, 2}}}+\frac{1}{\overline{\mathrm{K}_{i, 3}}}} \Leftrightarrow \overline{\mathrm{K}_{i, c}}=\frac{3 \overline{\mathrm{K}_{i, 1}} \cdot \overline{\mathrm{K}_{i, 2}} \cdot \overline{\mathrm{K}_{i, 3}}}{\overline{\mathrm{K}_{i, 2}}+\overline{\mathrm{K}_{i, 1}} \cdot \overline{\mathrm{K}_{i, 3}}+\overline{\mathrm{K}_{i, 2}} \cdot \overline{\mathrm{K}_{i, 3}}}
$$

